\title{
Model predictive control for multi-class traffic flows*
}

\author{
P. Deo, B. De Schutter, and A. Hegyi
}

If you want to cite this report, please use the following reference instead:

P. Deo, B. De Schutter, and A. Hegyi, "Model predictive control for multi-class traffic flows," Proceedings of the 12th IFAC Symposium on Transportation Systems, Redondo Beach, California, pp. 25-30, Sept. 2009.

Delft Center for Systems and Control Delft University of Technology

Mekelweg 2, 2628 CD Delft

The Netherlands

phone: +31-15-278.24.73 (secretary)

URL: https: / / www.dcsc.tudelft.nl

${ }^{*}$ This report can also be downloaded via https://pub.deschutter.info/abs/09_023.html 


\title{
Model Predictive Control for Multi-Class Traffic Flows
}

\author{
Puspita Deo ${ }^{*}$ Bart De Schutter ${ }^{* * *}$ Andreas Hegyi ${ }^{* * *}$ \\ ${ }^{*}$ Marine \& Transport Technology department, Delft University of \\ Technology, Delft, The Netherlands (email: p.deo@tudelft.nl) \\ ${ }^{* *}$ Delft Center for Systems and Control, Delft University of Technology, \\ Delft, The Netherlands (email: b@deschutter.info) \\ *** Department of Transport \& Planning, Faculty of Civil Engineering and \\ Geosciences, Delft University of Technology, Delft, The Netherlands (email: \\ a.hegyi@tudelft.nl)
}

\begin{abstract}
In this paper we first present an extension of the macroscopic traffic flow model METANET to multi-class flows. The resulting multi-class model takes into account the differences between, e.g., fast vehicles (cars) and slow vehicles (trucks) including their possibly different free-flow speeds and critical densities. Next, we show how this model can be used in a model-based predictive control approach for coordinated and integrated traffic flow control. In particular, we use Model Predictive Control (MPC) to coordinate various traffic control measures such as variable speed limits, ramp metering, etc. Using a simple benchmark example from the literature we illustrate that by taking the heterogeneous nature of multi-class traffic flows into account a better performance can be obtained.
\end{abstract}

Keywords: Heterogenous traffic flows, multi-class traffic, traffic models, model-based control, dynamic traffic management.

\section{INTRODUCTION}

Due to growing percentage of car ownership, and the increase in public transportation as well as transportation of goods, the capacity of most roads is frequently reached or even exceeded, resulting in frequent traffic congestion and leading to reduced throughput, excess delays, reduced safety, and environmental issues. The steadily increasing number and length of traffic jams on freeways has led to the use of several dynamic traffic management measures all over the world such as on-ramp metering, dynamic routing, and the provision of congestion information. In order to be able to coordinate the various control measures model-based control methods offer promising perspectives (Papageorgiou et al., 1990a,b; Hegyi, 2004). One essential feature of such approaches is the traffic flow model. In this paper we will use (an extended version of) the macroscopic traffic flow model METANET proposed in (Messmer and Papageorgiou, 1990; Kotsialos et al., 2002a, 1999b; Papageorgiou et al., 1990a). In particular, we will extend this model to heterogeneous, multi-class traffic flows and integrate it in a model-based control approach.

Most of the microscopic traffic flow models available today (such as Paramics, AIMSUN, CORSIM, VISSIM, etc.) are essentially multi-class. However, for on-line model-based control purposes microscopic models are in practice too slow and faster models are required. In this context, macroscopic traffic flow models offer an appropriate trade-off between speed of execution and accuracy. There exists a wide variety of macroscopic traffic flow models (Lighthill and Whitham, 1955a,b; Hoogendoorn and Bovy, 2001; Messmer and Papageorgiou, 1990; Daganzo, 1997). In their basic version these models were mostly non-heterogeneous. However, for some macroscopic traffic models multi-class versions have already been proposed such as multi-class extensions of the Lighthill-Whitham-Richards
(LWR) model (Logghe, 2003; Wong and Wong, 2002; BenzoniGavage and Colombo, 2003) or the Fastlane model (van Lint et al., 2008a,b). Nevertheless, the macroscopic models used for on-line traffic control purposes are mostly non-heterogeneous. Since the METANET model has been used extensively for model-based control by several authors (see, e.g., Messmer and Papageorgiou (1990); Kotsialos et al. (1999b, 2002b); Bellemans et al. (2006); Hegyi (2004); Hegyi et al. (2005b)) and since to the authors' best knowledge no multi-class extension of the METANET model has been described yet, we propose a multi-class version of the METANET model in this paper.

Once the multi-class METANET model has been described, we also discuss how it can be incorporated in a model-based control approach. In particular, we apply a model predictive control (MPC) framework (Camacho and Bordons, 1995; Maciejowski, 2002) to find the optimal combination of control measures (control inputs). MPC is an optimal control method applied in a rolling horizon framework. Optimal control has been successfully applied by Papageorgiou et al. (1990b); Kotsialos et al. (1999a,b, 2002b) to coordinate or to integrate traffic control measures. Both optimal control and MPC have the advantage that the controller generates control signals that are optimal according to a user-supplied objective function. However, MPC offers some important advantages over conventional optimal control. First, optimal control has an open-loop structure, which means that the disturbances (in our case: the traffic demands) have to be completely and exactly known before the simulation, and that the traffic model has to be very accurate to ensure sufficient precision for the whole simulation. MPC operates in closed-loop, which means that the traffic state and the current demands are regularly fed back to the controller, and the controller can take disturbances into account and correct for prediction errors resulting from model mismatch. Second, adaptivity is easily implemented in MPC, because the prediction model 


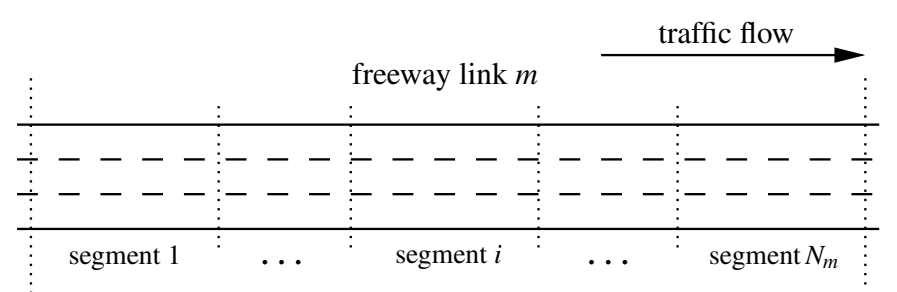

Fig. 1. In the METANET model a freeway link is divided into segments.

can be changed or replaced during operation. This may be necessary when traffic behavior significantly changes (e.g., in case of incidents, changing weather conditions, lane closures for maintenance). Third, for MPC a shorter prediction horizon is usually sufficient, which reduces complexity, and makes the real-time application of MPC feasible.

\section{ORIGINAL METANET MODEL}

In this section we briefly recapitulate the basic (destinationindependent) METANET model. We refer the interested reader to Messmer and Papageorgiou (1990); Kotsialos et al. (2002a, 1999b); Papageorgiou et al. (1990a) for a full description of the METANET model.

\subsection{Basic METANET model}

The METANET model represents a network as a directed graph with the links corresponding to freeway stretches. Each freeway link has uniform characteristics, i.e., no on-ramps or off-ramps, and no major changes in geometry. Where major changes occur in the characteristics of the link or in the road geometry (e.g., on-ramp or an off-ramp), a node is placed. Each link $m$ is divided into $N_{m}$ segments of length $L_{m}$ (see Fig. 1). The number of lanes of link $m$ is denoted by $\lambda_{m}$.

Each segment $i$ of each link $m$ of the network is characterized by the traffic density $\rho_{m, i}(k)(\mathrm{veh} / \mathrm{km} / \mathrm{lane})$, the space-mean speed $v_{m, i}(k)(\mathrm{km} / \mathrm{h})$, and the outflow $q_{m, i}(k)(\mathrm{veh} / \mathrm{h})$, all at time step $k$. Here, time step $k$ corresponds to the time instant $t=k T$, where $T$ is the time step used for the simulation of the traffic flow (typically $T=10 \mathrm{~s}$ ). In the METANET model the relations between these variables and their evolution are described by the following equations:

$$
\begin{aligned}
q_{m, i}(k) & =\rho_{m, i}(k) v_{m, i}(k) \lambda_{m} \\
\rho_{m, i}(k+1)=\rho_{m, i}(k) & +\frac{T}{L_{m} \lambda_{m}}\left(q_{m, i-1}(k)-q_{m, i}(k)\right) \\
v_{m, i}(k+1)=v_{m, i}(k) & +\frac{T}{\tau}\left(V\left(\rho_{m, i}(k)\right)-v_{m, i}(k)\right) \\
& +\frac{T}{L_{m}} v_{m, i}(k)\left(v_{m, i-1}(k)-v_{m, i}(k)\right) \\
& -\frac{\eta T}{\tau L_{m}} \frac{\rho_{m, i+1}(k)-\rho_{m, i}(k)}{\rho_{m, i}(k)+\kappa},
\end{aligned}
$$

where $\tau, \eta$ and $\kappa$ are model parameters, and where the "desired" speed $V$ is given by

$$
V\left(\rho_{m, i}(k)\right)=v_{\text {free }, m} \cdot \exp \left[-\frac{1}{a_{m}}\left(\frac{\rho_{m, i}(k)}{\rho_{\text {crit }, m}}\right)^{a_{m}}\right],
$$

with $a_{m}$ a model parameter, $v_{\text {free, } m}$ the free-flow speed, and $\rho_{\text {crit }, m}$ the critical density for link $m$.

Equation (1) describes the physical relation between the three state variables, (2) describes the conservation of vehicles, and
(3) characterizes the evolution of the speed, consisting of three update terms: a relaxation term that expresses that the drivers try to achieve a desired speed $V(\rho)$, a convection term that expresses the speed increase (or decrease) caused by the inflow of vehicles, and an anticipation term that expresses the speed decrease (or increase) as drivers experience a density increase (or decrease) downstream.

In addition, mainstream or on-ramp origins are modeled with a simple queue model: the length $w_{o}$ of the queue at origin $o$ evolves as follows:

$$
w_{o}(k+1)=w_{o}(k)+T\left(d_{o}(k)-q_{o}(k)\right),
$$

where $d_{o}(k)$ is the origin demand and $q_{o}(k)$ the outflow:

$$
q_{o}(k)=\min \left[d_{o}(k)+\frac{w_{o}(k)}{T}, Q_{o}\left(\frac{\rho_{\max , m}-\rho_{m, 1}(k)}{\rho_{\max , m}-\rho_{\text {crit }, m}}\right)\right],
$$

with $Q_{o}$ the mainstream or on-ramp origin capacity (veh/h) under free-flow conditions, and $\rho_{\max , m}$ (veh/ $\left./ \mathrm{km} / \mathrm{lane}\right)$ the $\max -$ imum density of the link $m$ to which the origin is connected.

The basic METANET model may also contain some extra merging terms (for on-ramps) as well as weaving terms (in case there is a reduction in the number of lanes). There are also node equations to model the connections between links like joins and splits. However, for the sake of brevity these equations are not given here explicitly. For details we refer to Messmer and Papageorgiou (1990); Kotsialos et al. (2002a, 1999b); Papageorgiou et al. (1990a). Moreover, Hegyi (2004); Hegyi et al. (2005b,a) have also proposed some additional extensions to model dynamic speed limits (see also Section 2.2 below), main-stream metering, density-dependent anticipation constants, and improved downstream boundary conditions.

\subsection{Effect of control actions}

The METANET model can also capture the effects of control measures. In view of the control measures discussed in the example of Section 5 we now present the equations for including the effects of ramp metering and of dynamic speed limits. For more details and for additional control measures we refer to Messmer and Papageorgiou (1990); Kotsialos et al. (2002a, 1999b); Papageorgiou et al. (1990a); Hegyi (2004); Hegyi et al. (2005a).

For ramp metering equation (5) can be extended as follows:

$$
\begin{aligned}
q_{o}(k)=\min \left[d_{o}(k)+\right. & \frac{w_{o}(k)}{T}, Q_{o} \cdot r_{o}(k), \\
& \left.Q_{o}\left(\frac{\rho_{\max , m}-\rho_{m, 1}(k)}{\rho_{\max , m}-\rho_{\mathrm{crit}, m}}\right)\right],
\end{aligned}
$$

where $r_{o}(k) \in[0,1]$ is the ramp metering rate at time step $k$.

For speed limits Hegyi (2004); Hegyi et al. (2005b,a) have proposed to replace (4) by the following equation:

$$
\begin{array}{r}
V\left(\rho_{m, i}(k)\right)=\min \left(v_{\text {free }, m} \cdot \exp \left[-\frac{1}{a_{m}}\left(\frac{\rho_{m, i}(k)}{\rho_{\text {crit }, m}}\right)^{a_{m}}\right],\right. \\
\left.(1+\alpha) v_{\text {control }, m, i}(k)\right),
\end{array}
$$

where $v_{\text {control, } m, i}(k)$ is the speed limit imposed on segment $i$ of link $m$ at time step $k$, and $1+\alpha$ is the non-compliance factor 
that expresses that drivers usually do not fully comply with the displayed speed limit and their target speed.

\section{MULTI-CLASS METANET MODEL}

Now we extend the basic METANET model to heterogeneous, multi-class traffic flows. We assume that there are $C$ different classes of vehicles present in the network. Each of these classes will be described by its own state variables. In order to take differences in the typical lengths of the vehicles for each class into account, we will express all variables in so-called "equivalent vehicles ${ }^{1}$ " (e.g., a truck could then count for three regular vehicles). In particular, the state of the traffic network will be described by

- the equivalent density fraction $\theta_{m, i, c}(k)$ of vehicles of class $c$ in segment $i$ of link $m$ at time step $k$,

- the equivalent partial traffic density $\rho_{m, i, c}(k)$ (veh/km/lane) for vehicles of class $c$ in segment $i$ of link $m$ at time step $k$,

- the space-mean speed $v_{m, i, c}(k)(\mathrm{km} / \mathrm{h})$ for vehicles of class $c$ in segment $i$ of link $m$ at time step $k$,

- the equivalent partial outflow $q_{m, i, c}(k)(\mathrm{veh} / \mathrm{h})$ for vehicles of class $c$ in segment $i$ of link $m$ at time step $k$.

Since we use equivalent vehicles, the relation between the actual densities and the equivalent partial densities can be expressed as follows: If we use class 1 as a base class, and if $\rho_{m, i, c}^{\text {actual }}(k)$ is the actual density of vehicles of class $c$ (i.e., the real, physical number of vehicles of class $c$ in segment $i$ of link $m$ at time step $k$ ), then the equivalent partial traffic density $\rho_{m, i, c}(k)$ is defined as

$$
\rho_{m, i, c}(k)=\frac{L_{c}^{\mathrm{veh}}}{L_{1}^{\mathrm{veh}}} \rho_{m, i, c}^{\mathrm{actual}}(k)
$$

where $L_{c}^{\mathrm{veh}}$ denotes the typical vehicle length for class $c$. A similar reasoning holds for the density fractions and the outflows.

Now we present the equations that describe the relations between the state variables and their evolution. First of all, we have

where

$$
\theta_{m, i, c}(k)=\frac{\rho_{m, i, c}(k)}{\rho_{m, i, \text { tot }}(k)}
$$

$$
\rho_{m, i, \text { tot }}(k)=\sum_{c=1}^{C} \rho_{m, i, c}(k)
$$

is the total equivalent density in segment $i$ of link $m$ at time step $k$.

Equations (1) and (2) also hold for the equivalent partial flows and densities:

$$
\begin{aligned}
q_{m, i, c}(k) & =\rho_{m, i, c}(k) v_{m, i, c}(k) \lambda_{m} \\
\rho_{m, i, c}(k+1) & =\rho_{m, i, c}(k)+\frac{T}{L_{m} \lambda_{m}}\left(q_{m, i-1, c}(k)-q_{m, i, c}(k)\right) .
\end{aligned}
$$

For the speed equation we have to make a distinction between the convection term, which depends on the (class-dependent) speed with which the vehicles leave the previous segment, and the anticipation term, which depends on the total density in the current and the next segment. This yields:

\footnotetext{
1 This is related to the concepts Passenger Car Unit (PCU) or Passenger Car Equivalent (PCE).
}

$$
\begin{aligned}
& v_{m, i, c}(k+1)=v_{m, i, c}(k)+ \\
& \frac{T}{\tau_{c}}\left(\widetilde{V}\left(\rho_{m, i, \text { tot }}(k), \theta_{m, i, 1}(k), \ldots, \theta_{m, i, C}(k), c\right)-v_{m, i, c}(k)\right) \\
& \quad+\frac{T}{L_{m}} v_{m, i, c}(k)\left(v_{m, i-1, c}(k)-v_{m, i, c}(k)\right) \\
& \quad-\frac{\eta_{c} T}{\tau_{c} L_{m}} \frac{\rho_{m, i+1, \text { tot }}(k)-\rho_{m, i, t o t}(k)}{\rho_{m, i, t o t}(k)+\kappa_{c}}
\end{aligned}
$$

For $\widetilde{V}$ there are several options: one is to use simple (convex) interpolation between the different fundamental diagrams for each class cut off at the desired speed of the given class:

$$
\begin{aligned}
& \widetilde{V}\left(\rho_{m, i, \text { tot }}(k), \theta_{m, i, 1}(k), \ldots, \theta_{m, i, C}(k), c\right)= \\
& \quad \min \left(V_{c}\left(\rho_{m, i, \text { tot }}(k)\right), \sum_{\gamma=1}^{C} \theta_{m, i, \gamma}(k) V_{\gamma}\left(\rho_{m, i, \text { tot }}(k)\right)\right)
\end{aligned}
$$

with $V_{c}$ given by (4) or (7), but with class-dependent parameters $v_{\text {free }, m, c}, a_{m, c}, \alpha_{c}$, and with the joint critical density $\rho_{\text {crit }, m}$, e.g.,

$$
V_{c}\left(\rho_{m, i, \text { tot }}(k)\right)=v_{\text {free }, m, c} \cdot \exp \left[-\frac{1}{a_{m, c}}\left(\frac{\rho_{m, i, \text { tot }}(k)}{\rho_{\text {crit }, m}}\right)^{a_{m, c}}\right]
$$

in case there is no speed limit active in link $m$. Equation (7) can be transformed in a similar way.

Alternatively, one could use the approach also used in the Fastlane model (van Lint et al., 2008a,b):

$$
\begin{aligned}
& \widetilde{V}\left(\rho_{m, i, \text { tot }}(k), \theta_{m, i, 1}(k), \ldots, \theta_{m, i, C}(k), c\right)= \\
& \left\{\begin{array}{l}
v_{\text {free }, m, c}-\frac{\rho_{m, i, \text { tot }}(k)}{\rho_{\text {crit }, m}}\left(v_{\text {free }, m, c}-v_{\text {crit }, m}\right) \text { if } \rho_{m, i, \text { tot }}(k)<\rho_{\text {crit }, m} \\
\frac{v_{\text {crit }, m} \rho_{m, i, \text { tot }}(k)}{\rho_{\text {crit }, m}}\left(1-\frac{\rho_{m, i, \text { tot }}(k)-\rho_{\text {crit }, m}}{\rho_{\text {max }, m}-\rho_{\text {crit }, m}}\right) \text { otherwise }
\end{array}\right.
\end{aligned}
$$

where $\rho_{\text {crit }, m}$ and $v_{\text {crit }, m}$ are respectively the joint critical density and joint critical speed for link $m, \rho_{\max , m}$ is the effective jam density for link $m$, and $\rho_{m, i}(k)$ is the total density in segment $i$ of link $m$ at time step $k$ (cf. (8)). So in this case the desired speed is equal for all classes when the total density is larger than the critical density, and below the critical density different classes move with different speed.

For each origin $o$ we also introduce partial queue lengths $w_{o, c}$ which evolve as follows:

$$
w_{o, c}(k+1)=w_{o, c}(k)+T\left(d_{o, c}(k)-q_{o, c}(k)\right),
$$

where $d_{o, c}(k)$ is the equivalent partial origin demand for class $c$ (expressed in equivalent vehicles per hour), and $q_{o, c}(k)$ the equivalent partial outflow, which is described by an extension of equation (5):

$q_{o, c}(k)=\min \left[q_{o, c}^{\mathrm{des}}(k), \frac{q_{o, c}^{\mathrm{des}}(k)}{\sum_{\gamma=1}^{C} q_{o, \gamma}^{\mathrm{des}}(k)} Q_{o}\left(\frac{\rho_{\mathrm{max}, m}-\rho_{m, 1, \mathrm{tot}}(k)}{\rho_{\mathrm{max}, m}-\rho_{\mathrm{crit}, m}}\right)\right]$

with

$$
q_{o, c}^{\mathrm{des}}(k)=d_{o, c}(k)+\frac{w_{o, c}(k)}{T}
$$

the desired origin outflow for class $c$ at origin $o$, and where the second argument of the min operator in (13) divides the available capacity over the different class in proportion to the desired origin outflows. Note that (6) can be adapted in a similar way.

\section{MODEL PREDICTIVE CONTROL TRAFFIC CONTROL}

To solve the problem of coordination of traffic control measures (and in particular dynamic speed limits and ramp metering 


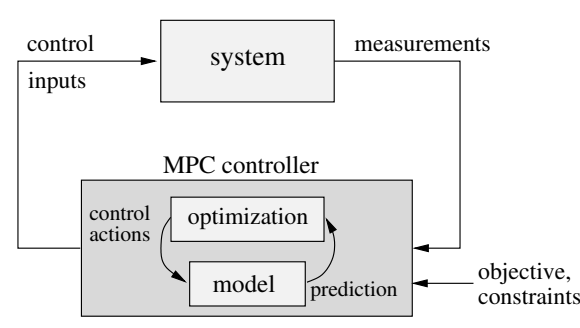

Fig. 2. Schematic view of the MPC structure.

- although the approach can easily be extended to include other control measures), we adopt the model predictive control (MPC) scheme (Camacho and Bordons, 1995; Maciejowski, 2002) (see Fig. 2). In the MPC control scheme a discrete-time model is used to predict the future behavior of the process, and the MPC controller uses (numerical) optimization to determine the control signals that result in an optimal process behavior over a given prediction horizon. The resulting optimal control inputs are applied using a rolling horizon scheme.

More specifically, at each control $\operatorname{step}^{2} k_{\mathrm{c}}$ (corresponding to time $t=T_{\mathrm{c}} k_{\mathrm{c}}$ where $T_{\mathrm{c}}$ is the control time step) the state of the traffic system is measured or estimated, and an optimization is performed over the prediction horizon $\left[k_{\mathrm{c}} T_{\mathrm{c}},\left(k_{\mathrm{c}}+N_{\mathrm{p}}\right) T_{\mathrm{c}}\right]$ to determine the optimal control inputs. Only the first value of the resulting control signal (the control signal for time step $k_{\mathrm{c}}$ ) is then applied to the process. At the next control step $k_{\mathrm{c}}+1$ this procedure is repeated.

For the sake of simplicity we assume in this paper that $T_{\mathrm{c}}$ is an integer multiple of $T$, i.e., $T_{\mathrm{c}}=M T$ with $M$ an integer.

Note that in our case the fractions of vehicles of each class will typically have to be measured using cameras, as conventional sensors such as induction loops can usually not easily discern between different vehicle classes. Alternatively, if only simple measurements are available that do not directly differentiate between different vehicle classes, state estimation methods have to be applied (see, e.g., (van Lint et al., 2008a)).

To reduce complexity and improve stability often a control horizon $N_{\mathrm{c}}\left(\leq N_{\mathrm{p}}\right)$ is introduced in MPC, and after the control horizon has been passed the control signal is taken to be constant. So there are two loops: the rolling horizon loop and the optimization loop inside the controller. The loop inside the controller of Fig. 2 is executed as many times as needed to find the optimal control signals at control step $k_{\mathrm{c}}$, for given $N_{\mathrm{p}}, N_{\mathrm{c}}$, traffic state, and expected demand. The loop connecting the controller and the traffic system is performed once for each control step $k_{\mathrm{c}}$ and provides the state feedback to the controller. This feedback is necessary to correct for (the ever present) prediction errors, and to provide disturbance rejection (compensation for unexpected traffic demand variations). The advantage of this rolling horizon approach is that it results in an on-line adaptive control scheme that allows us to take changes in the system or in the system parameters into account by regularly updating the model of the system.

The MPC framework requires an objective function that expresses the performance of the traffic network (as a function of a given control input). The objective function we will use in the benchmark example is total time spent (TTS) by the vehicles in

\footnotetext{
2 Since the simulation time $T$ used for the METANET model (typically $10 \mathrm{~s}$ ) is in general different from the control time step $T_{\mathrm{c}}$ (typically 1-5 min), we also use different time step counters for the METANET model $(k)$ and for MPC $\left(k_{\mathrm{c}}\right)$.
}

the network (note however that the MPC approach is generic so that other objective functions can easily be generated). Moreover, to get a smooth control signal we add a small term that penalizes abrupt changes in the control signal. In particular, the model predictive control algorithm finds the control values $^{3} R_{o}\left(k_{\mathrm{c}}+j\right)$ (ramp metering rates) and $V_{\text {control }, m, i}\left(k_{\mathrm{c}}+j\right)$ (dynamic speed limits) for $j=0, \ldots, N_{\mathrm{c}}-1$ that minimize the following objective function over the period $\left[k_{\mathrm{c}} T_{\mathrm{c}},\left(k_{\mathrm{c}}+N_{\mathrm{p}}\right) T_{\mathrm{c}}\right)$ :

$$
\begin{aligned}
J(k) & =T \sum_{j=k_{\mathrm{c}} M}^{\left(k_{\mathrm{c}}+N_{\mathrm{p}}\right) M-1} \sum_{c=1}^{C} \frac{L_{1}^{\mathrm{veh}}}{L_{c}^{\mathrm{veh}}}\left(\sum_{m, i} \rho_{m, i, c}(j) L_{m} \lambda_{m}+\sum_{o} w_{o, c}(j)\right) \\
+ & \sum_{j=k_{\mathrm{c}}}^{k_{\mathrm{c}}+N_{\mathrm{c}}-1}\left[a_{\text {ramp }} \sum_{o \in O_{\text {ramp }}}\left(R_{o}(j)-R_{o}(j-1)\right)^{2}\right. \\
& \left.+a_{\text {speed }} \sum_{(m, i) \in I_{\text {speed }}}\left(\frac{V_{\text {control }, m, i}(j)-V_{\text {control }, m, i}(j-1)}{v_{\text {free }, m, \text { max }}}\right)^{2}\right]
\end{aligned}
$$

where $a_{\text {ramp }}$ and $a_{\text {speed }}$ are non-negative weight parameters, $v_{\text {free }, m, \max }=\max _{c} v_{\text {free }, m, c}, O_{\text {ramp }}$ is the set of indexes $o$ of the on-ramps where ramp metering is present, and $I_{\text {speed }}$ is the set of pairs of indexes $(m, i)$ of the links and segments where speed control is present. Note that the factor $\frac{L_{1}^{\text {veh }}}{L_{c}^{\text {veh }}}$ in the first term on the right-hand side of (14) is required to transform the equivalent densities and queue lengths back into actual, physical densities and queue lengths.

In order to connect the time scale of the METANET simulation model and that of the MPC approach it should be noted that the control signals $R_{O}$ and $V_{\text {control, } m, i}$ are in fact updated every $T_{\mathrm{c}}$ time units and that they are taken to be constant within each control period $\left[k_{\mathrm{c}} T_{\mathrm{c}},\left(k_{\mathrm{c}}+1\right) T_{\mathrm{c}}\right)$. So if $k=M k_{\mathrm{c}}$ then the $r_{o}$ and $v_{\text {control, } m, i}$ signals used in (6) and (7) are defined by

$$
r_{o}(k+\ell)=R_{o}\left(k_{\mathrm{c}}\right), v_{\mathrm{control}, m, i}(k+\ell)=V_{\text {control }, m, i}\left(k_{\mathrm{c}}\right)
$$

for $\ell=0, \ldots, M-1$.

In conventional MPC heuristic tuning rules have been developed to select appropriate values for $N_{\mathrm{p}}$ and $N_{\mathrm{c}}$. For MPC-based traffic control these rules have to be adapted (Bellemans et al., 2006; Hegyi et al., 2005a): the prediction horizon $N_{\mathrm{p}}$ is selected to be about the typical travel time in the network, and for the control horizon $N_{\mathrm{c}}$ we will select a value that represents a tradeoff between the computational effort and the performance.

In general, the optimization problems resulting from MPCbased traffic control will be nonlinear and nonconvex, which implies that global or multi-start local optimization methods (Pardalos and Resende, 2002) are required such as multi-start sequential quadratic programming (SQP), pattern search, genetic algorithms, or simulated annealing.

\section{A BENCHMARK PROBLEM}

In order to illustrate the control framework presented above we will now apply it to a simple traffic network. As benchmark example we reconsider the example of Hegyi et al. (2005a) and we compare the results for MPC with a non-heterogeneous and with a heterogenous, multi-class traffic flow model. Note that whenever we talk about vehicles in the remainder of this

\footnotetext{
3 We differentiate between control signals expressed as a function of the control time step $k_{\mathrm{c}}$ (represented by capitals) and as a function of the simulation time step $k$ (represented by small letters). Their relation is given in (15).
} 


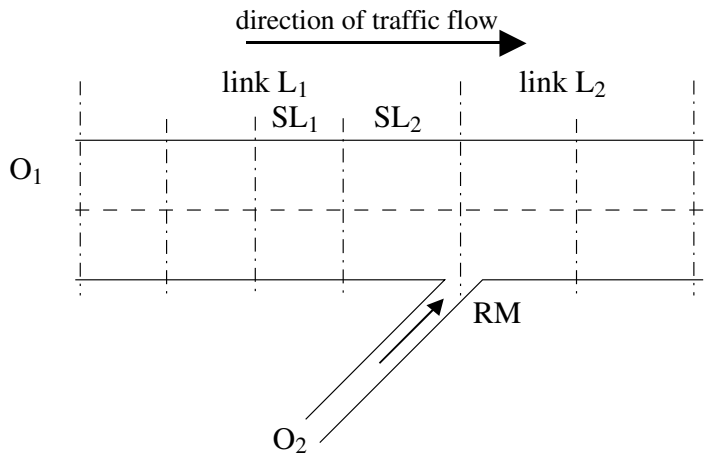

Fig. 3. Schematic representation of two-lane benchmark network consisting of two links $\mathrm{L}_{1}$ (with 4 segments) and Link $L_{2}$ (with two segments). Segments 3 and 4 of with $L_{1}$ are equipped with dynamic speed limits (marked by $\mathrm{SL}_{1}$ and $\left.\mathrm{SL}_{2}\right)$. There also is on on-ramp $\left(\mathrm{O}_{2}\right)$ equipped with ramp metering (marked by $\mathrm{RM})$. The traffic flow direction is from the left to the right.

section, we are in fact referring to "equivalent vehicles" as defined in Section 3. Alternatively, one could assume that the two vehicles considered in the example have the same nominal vehicle length.

\subsection{Set-up}

The benchmark network of Hegyi et al. (2005a) (see Fig. 3) consists of a mainstream freeway with two speed limits, and a metered on-ramp. The second speed limit is included to have more control over the state (speed, density) in the segment that is just before the on-ramp. The network considered consists of two origins (a mainstream and an on-ramp), two freeway links, and one destination. $\mathrm{O}_{1}$ is the main origin and has two lanes with a capacity of $2100 \mathrm{veh} / \mathrm{h}$ each. The freeway link $\mathrm{L}_{1}$ has two lanes, and is $4 \mathrm{~km}$ long consisting of four segments of $1 \mathrm{~km}$ each. Segments 3 and 4 are equipped with a variable message sign (VMS) where speed limits can be displayed. At the end of $\mathrm{L}_{1}$ a single-lane on-ramp $\left(\mathrm{O}_{2}\right)$ with a capacity of $2000 \mathrm{veh} / \mathrm{h}$ is attached. Link $\mathrm{L}_{2}$ follows with two lanes and two segments with length of $1 \mathrm{~km}$ each, and ends in destination $\mathrm{D}_{1}$, which has an unrestricted outflow. We assume that the queue length at $\mathrm{O}_{2}$ may not exceed 100 vehicles, in order to prevent spill-back to a surface street intersection.

As nominal network parameters we use the ones also used in Hegyi et al. (2005a) and Kotsialos et al. (1999b) ${ }^{4}: T=10 \mathrm{~s}$, $L=1 \mathrm{~km}, \tau=18 \mathrm{~s}, \kappa=40 \mathrm{veh} / \mathrm{km} /$ lane, $\eta=60 \mathrm{~km}^{2} / \mathrm{h}, \rho_{\max }=$ $180 \mathrm{veh} / \mathrm{km} / \mathrm{lane}, \delta=0.0122, \alpha=0.1, v_{\text {free }, m}=102 \mathrm{~km} / \mathrm{h}$, $a_{m}=1.867, \rho_{\text {crit }, m}=33.5 \mathrm{veh} / \mathrm{km} /$ lane for $m=1,2$.

Moreover, we consider two vehicle classes with the following parameters: $L_{1}^{\mathrm{veh}}=L_{2}^{\mathrm{veh}}, v_{\text {free }, m, 1}=110 \mathrm{~km} / \mathrm{h}, a_{m, 1}=1.8, \alpha_{1}=$ 0.12 , and $\rho_{\text {crit }, m, 1}=30 \mathrm{veh} / \mathrm{km} / \mathrm{lane}$ for $m=1,2$. The parameters $v_{\text {free, } m, 2}, a_{m, 2}, \alpha_{2}$, and $\rho_{\text {crit } m, 2}$ for class 2 are selected in such a way that the nominal parameters defined above correspond to $70 \%$ vehicles of class 1 (i.e., $v_{\text {free }, m}=0.7 v_{\text {free }, m, 1}+0.3 v_{\text {free }, m, 2}$, etc.). The other parameters are equal to the nominal ones.

Just like in (Hegyi et al., 2005a) we consider a total simulation period of $2.5 \mathrm{~h}$ and we select $T_{\mathrm{c}}=1 \mathrm{~min}, N_{\mathrm{p}}=7, N_{\mathrm{c}}=5$,

4 The factor $\delta$ mentioned here is used in the expression for the on-ramp merging term, see (Messmer and Papageorgiou, 1990; Kotsialos et al., 2002a, 1999b; Papageorgiou et al., 1990a) for details.

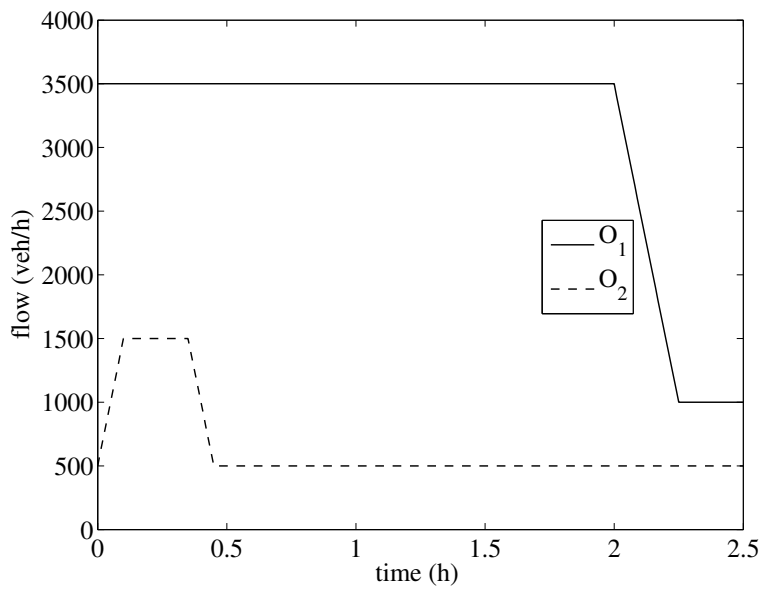

Fig. 4. The demand scenario considered in the simulation experiments.

\begin{tabular}{|c|c|c|c|}
\hline$\theta_{1}$ & no control & non-heterogenous MPC & multi-class MPC \\
\hline 0.9 & 1532.6 & 1347.8 & 1250.4 \\
0.7 & 2299.4 & 2206.8 & 2207.3 \\
0.5 & 2816.2 & 2758.2 & 2754.7 \\
0.3 & 3143.4 & 3103.9 & 3090.2 \\
0.1 & 3307.1 & 3276.4 & 3258.3 \\
\hline
\end{tabular}

Table 1. The TTS (veh.h) for the various scenarios.

$a_{\text {ramp }}=a_{\text {speed }}=0.4$, and the demand profile given in Fig. 4: The mainstream origin demand has a constant, relatively high level and a drop after 2 hours to a low value in 15 minutes. The demand on the on-ramp increases to near capacity, remains constant for 15 minutes, and decreases finally to a constant low value.

\subsection{Scenarios}

We will now simulate five possible input scenarios and three control scenarios. The input scenarios are characterized by different (constant) percentages of class 1 vehicles in the demand with $\theta_{1}$ at the origin and the on-ramp ranging in the set $\{0.1,0.3,0.5,0.7,0.9\}$. For the control scenarios we consider

- no control, i.e., the speed limits and the ramp metering are inactive (or equivalently, set to their maximum values),

- non-heterogenous MPC, i.e., in the prediction model we always take the nominal values for the model parameters (corresponding to a fixed value of $\theta_{1}=0.7$ ),

- heterogenous, multi-class MPC, i.e., for the prediction model we use the multi-class METANET model of Section 3 , with the real values of $\theta_{1}$ at the origin and the onramp being considered.

In all three cases the multi-class METANET model of Section 3 is used as simulation model. Moreover, we have used equation (12) for the desired speed $\widetilde{V}$ in both the multi-class prediction model and in the simulation model.

\subsection{Results}

The results for the various scenarios are displayed in Table 1. The TTS listed in the table corresponds to the total time spent over the entire simulation period of $2.5 \mathrm{~h}$. The MPC cases correspond to closed-loop operation where MPC is applied in a 
moving horizon approach. These results show that not taking the heterogeneity of the traffic flows into account leads to a degraded performance (note that for $\theta=0.7$, where one would expect the same value for both cases, the small difference in the results is due to the effects of the random choice of the initial starting points in the multi-start SQP optimization).

\section{CONCLUSIONS}

We have extended the original METANET traffic flow model to the case of multi-class traffic flows. In particular, for each class of vehicles partial (equivalent) states were introduced and adapted METANET equations for their relation and evolution were proposed. Next, we have applied the proposed model in a model predictive control approach to traffic networks with variable speed limits and ramp metering. This idea was illustrated by a simple example network from literature for which we have compared the non-heterogenous and the heterogenous, multiclass control approach. with the total time spent in the network as performance measure. From the simulation experiments we can conclude that taking into account the heterogenous nature of the traffic flows can substantially improve the network performance.

Topics for future research include: further extension and refinement of the model (e.g., extending the approach that was derived in Logghe (2003) for the LWR model to the METANET model), model validation against real traffic data, and comparison with other (multi-class) traffic flow models.

\section{ACKNOWLEDGEMENTS}

Research supported by the Faculty of Mechanical, Maritime, and Materials Engineering of Delft University of Technology, the BSIK projects "Transition to Sustainable Mobility (TRANSUMO)" and "Next Generation Infrastructures (NGI)", the European COST Action TU0702, the European Network of Excellence HYCON, the Transport Research Center Delft, and the Delft Research Center Next Generation Infrastructures.

\section{REFERENCES}

T. Bellemans, B. De Schutter, and B. De Moor. Model predictive control for ramp metering of motorway traffic: A case study. Control Engineering Practice, 14(7):757-767, July 2006.

S. Benzoni-Gavage and R.M. Colombo. An n-populations model for traffic flow. European Journal of Applied Mathematics, 14:587-612, 2003.

E.F. Camacho and C. Bordons. Model Predictive Control in the Process Industry. Springer-Verlag, Berlin, Germany, 1995.

C.F. Daganzo. Fundamentals of Transportation and Traffic Operations. Pergamon, 1997. ISBN 0-08-042785-5.

A. Hegyi. Model Predictive Control for Integrating Traffic Control Measures. $\mathrm{PhD}$ thesis, Delft University of Technology, Delft, The Netherlands, February 2004. URL http://www . hegyi.info/thesis/dep_hegyi_ 20040203 .pdf. TRAIL Thesis Series T2004/2.

A. Hegyi, B. De Schutter, and H. Hellendoorn. Model predictive control for optimal coordination of ramp metering and variable speed limits. Transportation Research Part C, 13 (3):185-209, June 2005a.

A. Hegyi, B. De Schutter, and J. Hellendoorn. Optimal coordination of variable speed limits to suppress shock waves.
IEEE Transactions on Intelligent Transportation Systems, 6 (1):102-112, March 2005b.

S.P. Hoogendoorn and P.H.L. Bovy. State-of-the-art of vehicular traffic flow modelling. Proceedings of the Institution of Mechanical Engineers, Part I: Journal of Systems and Control Engineering, 215(4):283-303, August 2001.

A. Kotsialos, M. Papageorgiou, and A. Meßmer. Integrated optimal control of motorway traffic networks. In Proceedings of the 1999 American Control Conference (ACC'99), pages 2183-2187, San Diego, California, June 1999a.

A. Kotsialos, M. Papageorgiou, and A. Messmer. Optimal coordinated and integrated motorway network traffic control. In Proceedings of the 14th International Symposium of Transportation and Traffic Theory (ISTTT), pages 621-644, Jerusalem, Israel, July 1999b.

A. Kotsialos, M. Papageorgiou, C. Diakaki, Y. Pavlis, and F. Middelham. Traffic flow modeling of large-scale motorway networks using the macroscopic modeling tool METANET. IEEE Transactions on Intelligent Transportation Systems, 3(4):282-292, December 2002a.

A. Kotsialos, M. Papageorgiou, M. Mangeas, and H. HajSalem. Coordinated and integrated control of motorway networks via non-linear optimal control. Transportation Research Part C, 10(1):65-84, February 2002b.

M.J. Lighthill and G.B. Whitham. On kinematic waves: I. Flood movement in long rivers. Proceedings of the Royal Society of London, 299A:281-316, May 1955a.

M.J. Lighthill and G.B. Whitham. On kinematic waves: II. A theory of traffic flow on long crowded roads. Proceedings of the Royal Society of London, 299A:317-345, May 1955b.

S. Logghe. Dynamic Modeling of Heterogeneous Vehicular Traffic. PhD thesis, Faculty of Applied Sciences, K.U.Leuven, Leuven, Belgium, June 2003.

J.M. Maciejowski. Predictive Control with Constraints. Prentice Hall, Harlow, England, 2002. ISBN 0-201-39823-0.

A. Messmer and M. Papageorgiou. METANET: A macroscopic simulation program for motorway networks. Traffic Engineering and Control, 31(9):466-470, 1990.

M. Papageorgiou, J.M. Blosseville, and H. Hadj-Salem. Modelling and real-time control of traffic flow on the southern part of Boulevard Périphérique in Paris: Part I: Modelling. Transportation Research Part A, 24(5):345-359, September 1990a.

M. Papageorgiou, J.M. Blosseville, and H. Haj-Salem. Modelling and real-time control of traffic flow on the southern part of Boulevard Périphérique in Paris: Part II: Coordinated on-ramp metering. Transportation Research Part A, 24(5): 361-370, September 1990b.

P.M. Pardalos and M.G.C. Resende, editors. Handbook of Applied Optimization. Oxford University Press, Oxford, UK, 2002. ISBN 0-19-512594-0.

J.W.C. van Lint, S.P. Hoogendoorn, and A. Hegyi. Dual EKF state and parameter estimation in multi-class first-order traffic flow models. In Proceedings of the 17th IFAC World Congress, pages 14078-14083, Seoul, Korea, July 2008a.

J.W.C. van Lint, S.P. Hoogendoorn, and M. Schreuder. Fastlane - a new multi-class first order traffic flow model. In Proceedings of the 87th Annual Meeting of the Transportation Research Board, Washington, DC, January 2008b. CD ROM.

G.C.K. Wong and S.C. Wong. A multi-class traffic flow model - an extension of LWR model with heterogeneous drivers. Transportation Research Part A, 36:(9):827-841, 2002. 